\title{
Heavy Flavour at the LHC
}

\section{Valerie Gibson}

Cavendish Laboratory, Department of Physics, University of Cambridge, J J Thomson Avenue, Cambridge, CB3 0DH, UK

E-mail: gibson@hep.phy.cam.ac.uk

\begin{abstract}
Heavy flavour physics provides a crucial role in the validation of the Standard Model of particle physics and in the search for new phenomena beyond. This review provides a personal summary of the headline results as of May 2013 from the LHC heavy flavour community in the subject areas of heavy flavour production and spectroscopy, mixing and $\mathrm{CP}$ violation and rare decays.
\end{abstract}




\section{Introduction}

In the lands of the North, where the Black Rocks stand guard against the cold sea, in the dark night that is very long, the people of the Northlands sit by their great log fires and they tell a tale of $₫$..... Heavy Flavour at the $L H C .$.

The physics of quarks and their interactions plays a crucial role in the validation of the Standard Model (SM) of particle physics and in the search for new phenomena beyond. At the Large Hadron Collider (LHC), the focus of quark flavour physics lies in the bottom and charm quark systems, which are sensitive, through quantum loop processes, to energy scales well beyond the centre-of-mass energy of the LHC. This socalled "heavy flavour" physics provides enormous potential for the discovery of physics beyond the SM and to distinguish between various new physics scenarios.

The SM description of the interactions between the different quark flavours is provided by the Cabibbo-Kobayashi-Maskawa (CKM) quark-mixing matrix [1], a fundamental consequence of the Yukawa couplings and electroweak symmetry breaking. The CKM theory is highly predictive, describing a huge range of phenomena with only four parameters; it is hierarchical, taking into account the full range of quark masses; and it accommodates matter-antimatter effects, via CP violation, with a single complex phase. The state-of-the-art knowledge of the CKM picture is encapsulated in the so-called "unitarity triangle" [2, 3], shown in Fig. 1] The experimental measurements accumulated over many decades, most recently by the BaBar 44 and Belle [5] experiments at the asymmetric $e^{+} e^{-}$colliders and the CDF [6] and D0 [7] experiments at the Tevatron $p \bar{p}$ collider, combined with theoretical input from Lattice QCD [8], show impressive consistency and a beautiful validation of the CKM picture.

The success of the CKM picture, however, implies that any new physics scenario must have a very particular flavour structure that does not give rise to inconsistences with SM expectations. To resolve this dilemma, the "minimal flavour violation" (MFV) hypothesis 9] proposes that all sources of flavour and CP violation in the quark sector have the same CKM pattern as in the SM. The power of heavy flavour physics therefore lies in those observables that are sensitive to new physics contributions and have an ability to prove or exclude the MFV hypothesis.

The LHC is currently the world's most copious source of $b$ and $c$ hadrons whose decays are selected and reconstructed by the LHC experiments. The LHCb detector [10] is a single-arm forward spectrometer, which runs at an instantaneous luminosity of $\sim 4 \times 10^{32} \mathrm{~cm}^{-2} \mathrm{~s}^{-1}$ and is designed specifically for the study of particles containing $b$ or $c$ quarks. The general purpose detectors, ATLAS [1] and CMS [12], although optimised to search for direct signatures of new particles, also exploit their capabilities in the heavy quark sector, particularly in b-hadron decays involving muons in the final state. Prior to the first LHC long-shutdown in 2012, the LHC experiments collected data corresponding to integrated luminosities of $\sim 25 \mathrm{fb}^{-1}$ (ATLAS/CMS) and $\sim 3 \mathrm{fb}^{-1}$

$\ddagger$ Taken from The Sagas of Noggin the Nog by Oliver Postgate. 


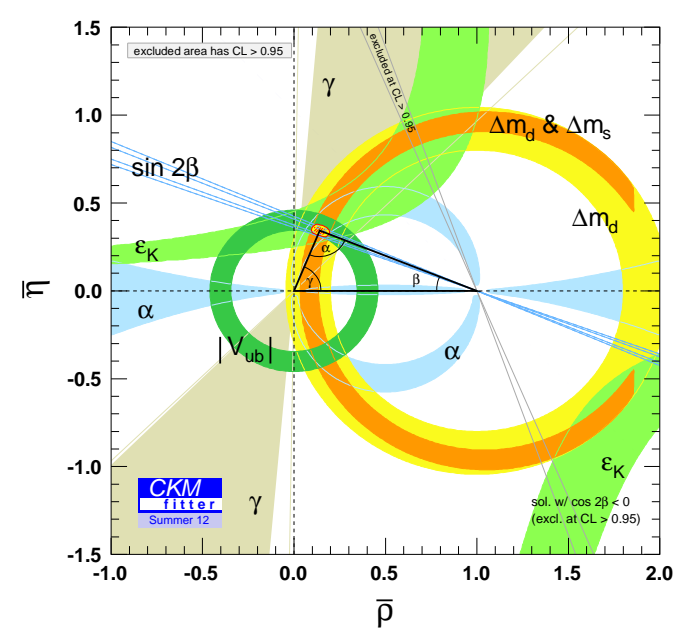

Figure 1. Constraints on the unitarity triangle in the $(\bar{\rho}, \bar{\eta})$ plane, taken from Ref. [2].

(LHCb). This review provides a personal summary of the headline results as of May 2013 from the LHC heavy flavour community in the subject areas of production and spectroscopy, mixing and $\mathrm{CP}$ violation and rare decays.

\section{Production and spectroscopy}

The LHC provides an excellent opportunity to study the strong interaction at high energies and resolve open puzzles in quantum chromodynamics. Open heavy flavour production, illustrated by the measurements of $B^{+}$meson $\$$ [13 and prompt charm production [14], shown in Fig. 2, is successfully described by well-developed and reliable tools, such as fixed-order with next-to-leading log summations (FONLL) and next-toleading order with parton showering (POWHEG or MC@NLO) calculations (an overview is given in Ref. [15]). The theory is less precise at describing onia production, such as the energy dependence of $J / \psi$ production [16], and stubbornly refuses to predict onia polarization, such as that of the $\Upsilon(\mathrm{nS})$ [1] as shown in Fig. 3]

The LHC experiments have collected an impressive catalogue of new and excited heavy flavour states in their first two years of operation, as well as first time observations of many new $B_{s}^{0}$ and $B_{c}^{+}$decay modes. Some highlights, shown in Fig. 团 include the discoveries of a new $\chi_{b}$ state by ATLAS [18], a new $\Xi_{b}$ state by CMS [19], and excited $\Lambda_{b}^{0}$ states by $\mathrm{LHCb}[20$. The experiments also measure masses and lifetimes with unrivalled precision. In particular, the $D$ meson mass differences [21] and the $\Lambda_{b}^{0}$ lifetime [22], where the tension with expectation observed previously is greatly reduced, are shown in Fig. 5 .

The exotic $X(3872)$ state, discovered in 2003 by the Belle experiment in $e^{+} e^{-}$ collisions using prompt $X(3872) \rightarrow J / \psi \pi^{+} \pi^{-}$decays 24, has also been observed at the LHC in $p p$ collisions [25, 26]. Although its $\mathrm{J}^{\mathrm{PC}}$ quantum numbers were limited to the values of either $1^{++}$or $2^{-+}$through an angular analysis of the prompt decays by the CDF

$\S$ Charge conjugate decay modes are implied throughout this review. 

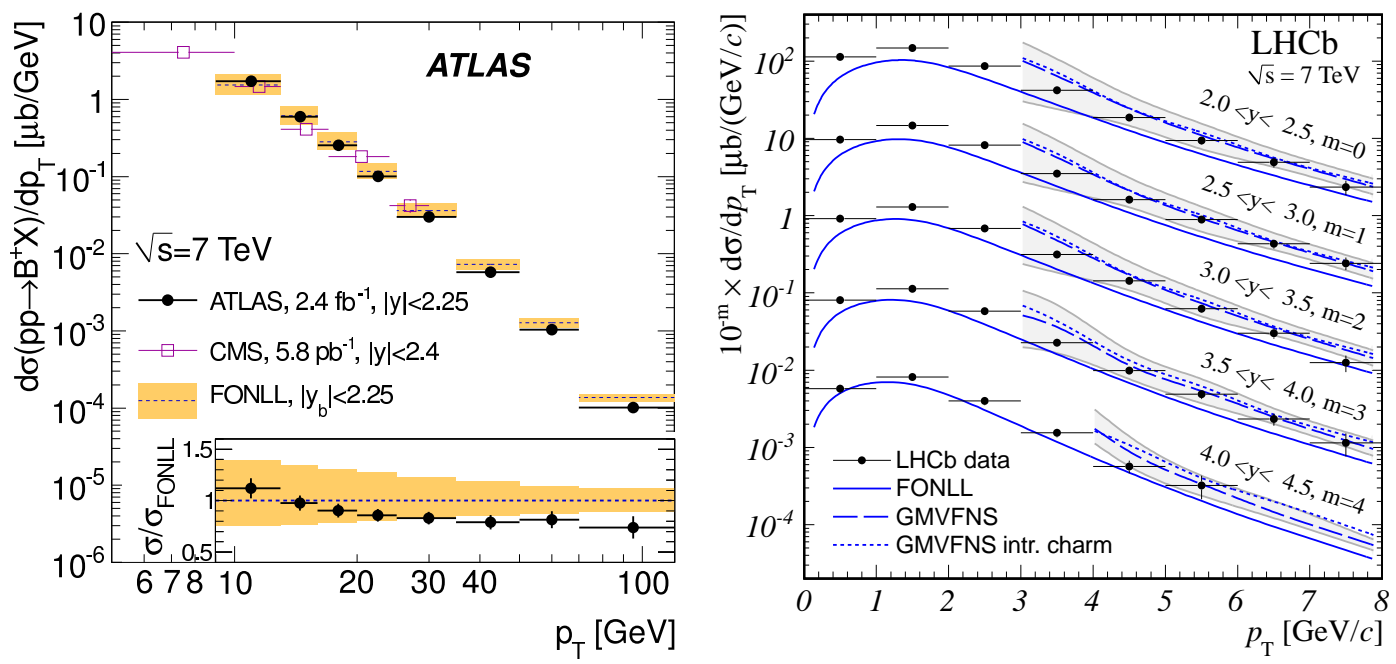

Figure 2. Differential cross-section versus $p_{\mathrm{T}}$ for (left) $B^{+}$13] and (right) $D^{0}$ 14 meson production, compared to theoretical predictions.
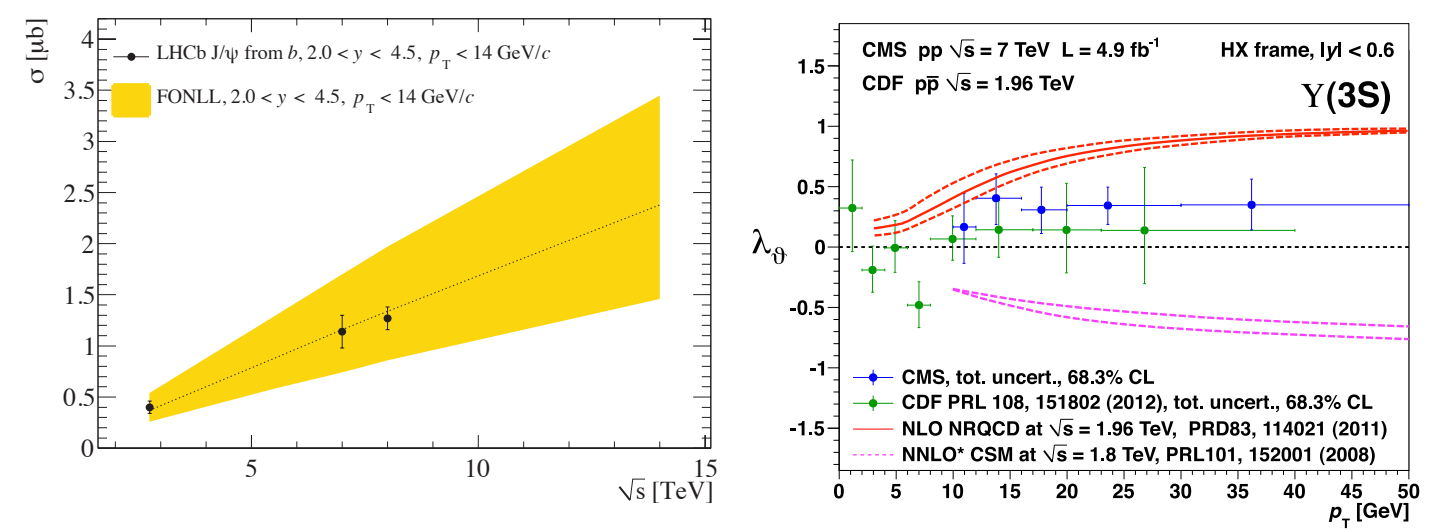

Figure 3. Comparison of data with theoretical predictions for (left) the production cross-section of $J / \psi$ from b-hadron decays as a function of centre-of-mass energy [16], and (right) the polarization parameter $\lambda_{\theta}$ for $\Upsilon(3 S)$ meson production as a function of $p_{\mathrm{T}}$ [17].
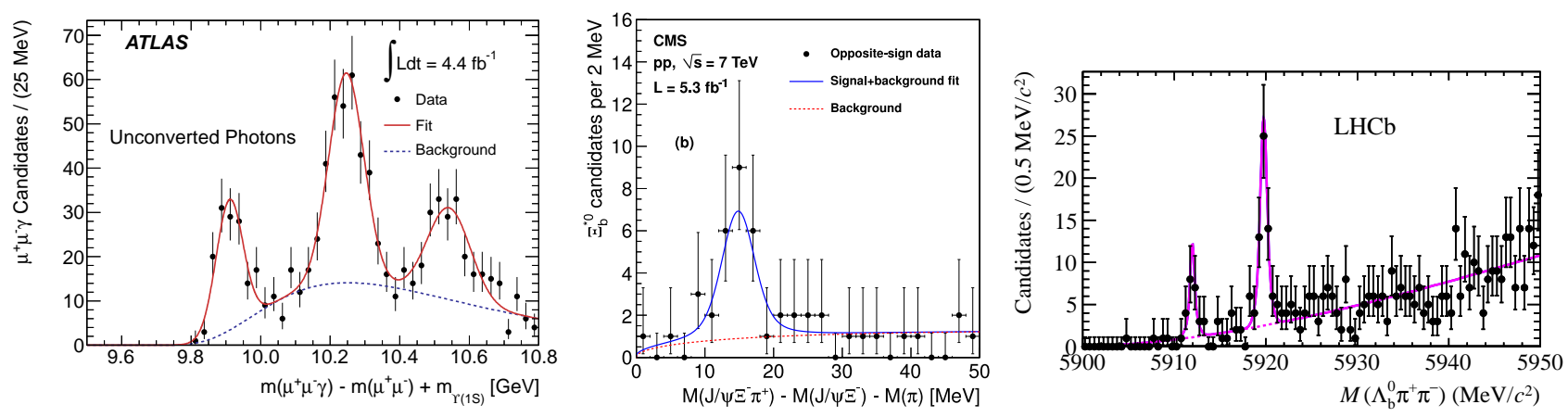

Figure 4. Mass distributions for the discovery of (left) a new $\chi_{b}$ state in $\chi_{b} \rightarrow \Upsilon(1 S) \gamma$ decays [18, (centre) a new $\Xi_{b}$ state in $\Xi_{b} \rightarrow \Xi_{b}^{-} \pi^{+}$, with $\Xi_{b}^{-} \rightarrow J / \psi \Xi^{-} \rightarrow \mu^{+} \mu^{-} \Lambda \pi^{-}$ and $\Lambda \rightarrow p \pi^{-}$decays [19], and (right) excited $\Lambda_{b}^{0}$ states in the $\Lambda_{b}^{0} \pi^{+} \pi^{-}$spectrum [20]. 

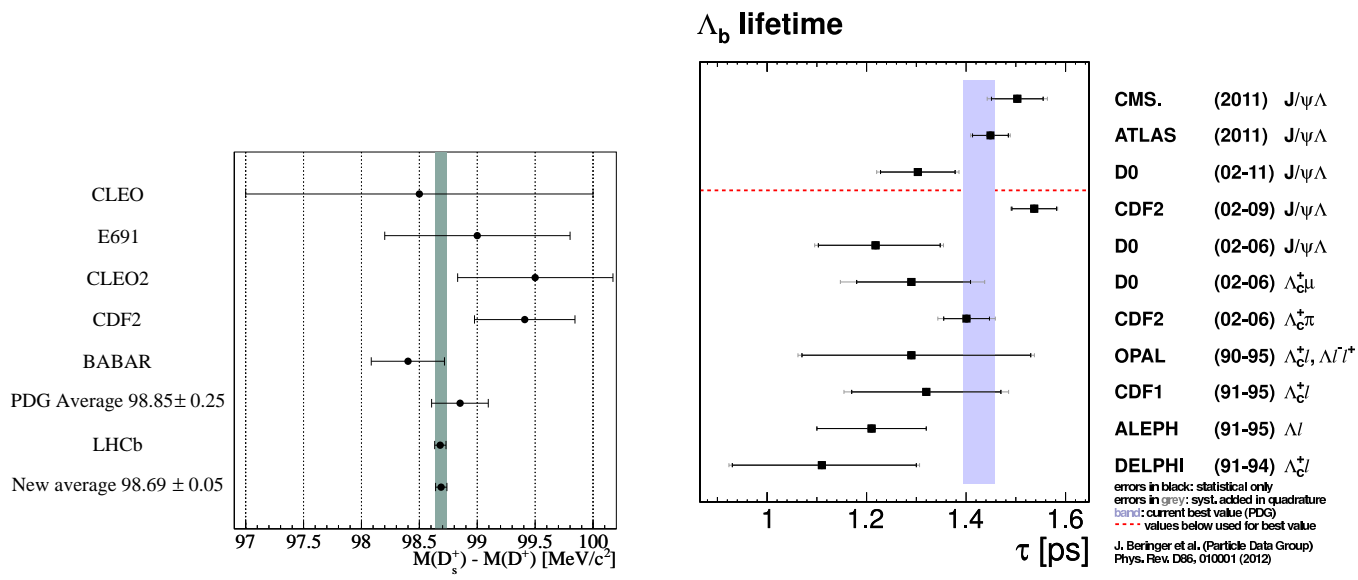

Figure 5. Measurements of (left) the $D_{s}^{+}-D^{+}$mass difference [21] and averages performed using the prescription given in Ref. [23], and (right) the $\Lambda_{b}^{0}$ lifetime [22].
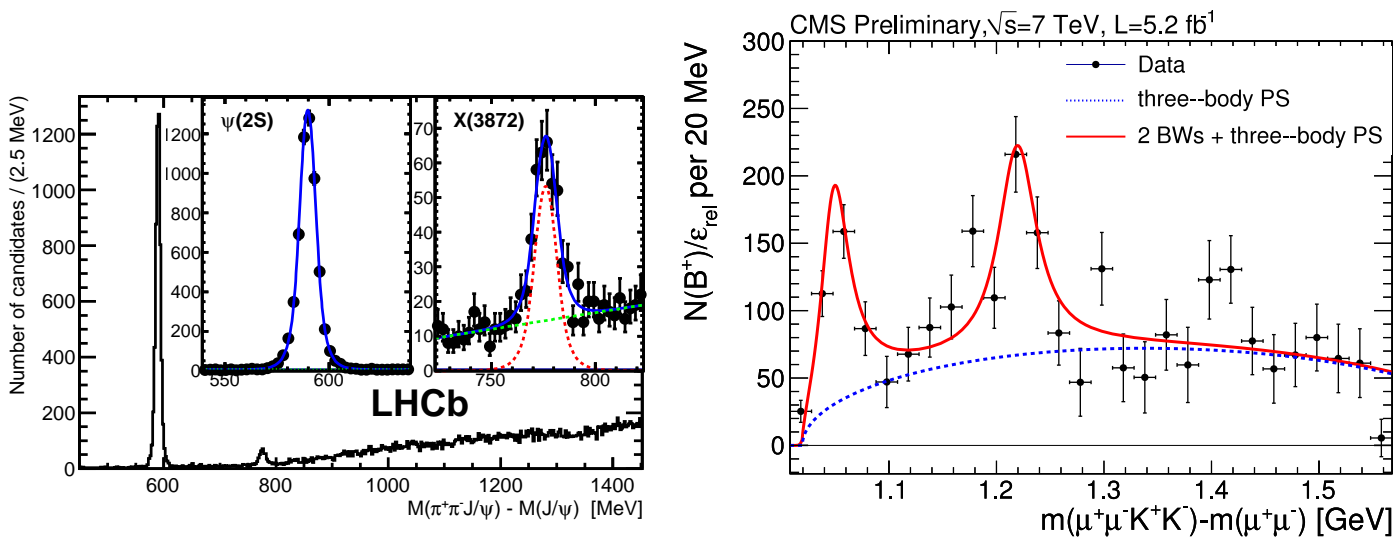

Figure 6. Distributions of (left) $M\left(J / \psi \pi^{+} \pi^{-}\right)-M(J / \psi)$ for $B^{+} \rightarrow J / \psi K^{+} \pi^{+} \pi^{-}$ candidates 28]; the fits of the $\psi(2 S)$ and $X(3872)$ signals are displayed, and (right) $M\left(\mu^{+} \mu^{-} K^{+} K^{-}\right)-M\left(\mu^{+} \mu^{-}\right)$for $B^{+} \rightarrow J / \psi \phi K^{+}$candidates 31 .

collaboration [27, its nature remained unclear since it did not fit well with conventional charmonium models. The LHCb collaboration has recently performed an analysis of the angular distributions in $B^{+} \rightarrow X(3872) K^{+}$decays, shown in Fig. 6(left), and have measured the $\mathrm{J}^{\mathrm{PC}}$ of the $X(3872)$ to be $1^{++}$[28], supporting a more unconventional $D \bar{D}^{*}$ molecular interpretation. The experiments are also in the process of searching for other new exotic states, such as the $X(4140)$, first observed by the CDF collaboration in $B^{+} \rightarrow J / \psi \phi K^{+}$decays [29], not observed by LHCb with first data [30], but recently confirmed by CMS 31, as shown in Fig. 6(right).

\section{Mixing and $\mathrm{CP}$ violation}

A major unsolved scientific mystery is the observation that our Universe consists entirely of matter (and no antimatter). One requirement to produce such a universe is the phenomenum of CP violation, well-established by experiment but with a magnitude insufficient to explain the observed baryon asymmetry in the Universe. New sources of 

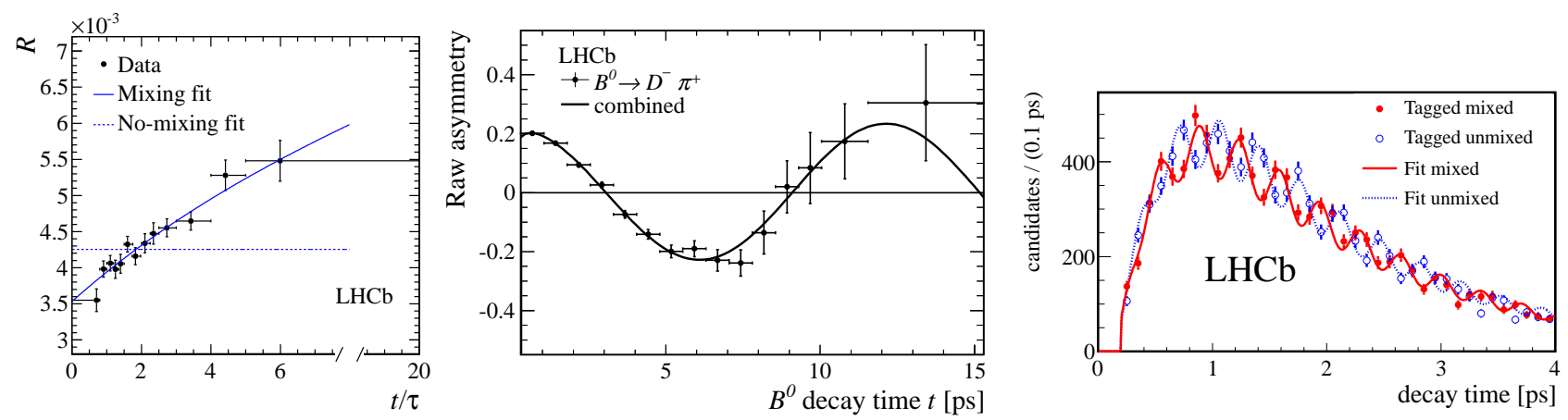

Figure 7. Decay time evolution of (left) the ratio of wrong-sign (mixed) $D^{0} \rightarrow K^{+} \pi^{-}$ to right-sign (unmixed) $D^{0} \rightarrow K^{-} \pi^{+}$in prompt $D^{*+} \rightarrow D^{0} \pi^{+}$decays 32, (centre) the mixing asymmetry $\left(N_{\text {mix }}-N_{\text {unmixed }}\right) /\left(N_{\text {mix }}+N_{\text {unmixed }}\right)$ for $B^{0} \rightarrow D^{-} \pi^{+}$decays [33, and (right) $B_{s}^{0}$ candidates tagged as mixed (red) and unmixed (blue) in $B_{s}^{0} \rightarrow D_{s}^{-} \pi^{+}$ decays [34].

$\mathrm{CP}$ violation must therefore exist, although the energy scale is unknown and there is no guarantee that they will be revealed in either the quark or neutrino sectors. The LHC experiments are therefore performing precision measurements of $\mathrm{CP}$ violation, and the related neutral meson mixing, in heavy quark systems to search for new phenomena that might shed light on this dilemma.

The phenomenon of a neutral meson mixing with its own antiparticle, described by box diagrams in the SM, has now been observed with oscillation frequencies over four orders of magnitude. Most recently, $D^{0}-\bar{D}^{0}$ mixing has been observed with a significance of 9.1 standard deviations by the LHCb collaboration using $D^{0}$ mesons, whose flavour at production is inferred from the charge of the pion in $D^{*+} \rightarrow D^{0} \pi^{+}$ decays [32]. The world's most precise measurements of the $B^{0}$ and $B_{s}^{0}$ oscillation frequencies, $\Delta m_{d}=0.5156 \pm 0.0051$ (stat) \pm 0.0033 (syst) $\mathrm{ps}^{-1}$ [33] and $\Delta m_{s}=$ $17.768 \pm 0.023$ (stat) \pm 0.006 (syst) $\mathrm{ps}^{-1}$ [34], have also been measured by LHCb using $B^{0} \rightarrow D^{-} \pi^{+}$and $B_{s}^{0} \rightarrow D_{s}^{-} \pi^{+}$decays, respectively. Examples of the measurements showing $D^{0}, B^{0}$ and $B_{s}^{0}$ mixing are given in Fig. [7. The measurements are still limited by their statistical precision and we therefore look forward to more precise measurements in the future.

Large CP violation effects in the $B^{0}$ system are well-established experimentally. In the SM, the CP-violating asymmetry arises from the interference between the amplitudes describing neutral meson mixing and the decay into the final state. This "mixinginduced" CP violation in the $B^{0}$ system was first discovered by the BaBar and Belle experiments through the measurement of the unitarity triangle observable $\sin 2 \beta$ using $B^{0} \rightarrow J / \psi K_{s}^{0}$ decays 35. This measurement is relatively theoretically clean although, as statistics increase at the LHC, the contribution from penguin diagrams, which can be studied in $B_{s}^{0} \rightarrow J / \psi K_{s}^{0}$ decays [36, will need to be taken into account.

The equivalent decay mode in the $B_{s}^{0}$ system, which also probes mixing-induced $\mathrm{CP}$ violation, is the theoretically-clean $B_{s}^{0} \rightarrow J / \psi \phi$ decay mode. Here, the SM CP- 


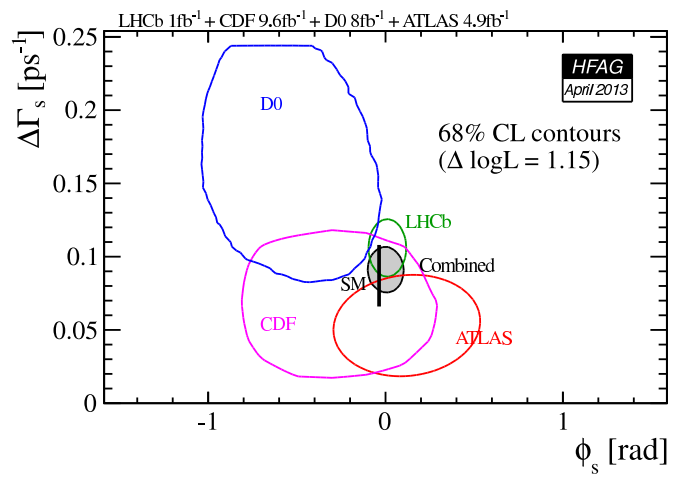

Figure 8. Measurements of the CP-violating phase $\phi_{s}$ versus the differences in decay widths of the $B_{s}^{0}$ mass eigenstates in resonant and non-resonant $B_{s}^{0} \rightarrow J / \psi K^{+} K^{-}$ (and $B_{s}^{0} \rightarrow J / \psi \pi^{+} \pi^{-}$) decays from CDF 37, D0 38, LHCb 39] and ATLAS [40]. The shaded region shows the combination of measurments and the thick line the SM prediction.

violating phase is well determined and very small, $\phi_{s}^{S M}=-0.0363 \pm 0.0017 \mathrm{rad}$. However, possible new physics in the box diagrams could substantially modify this phase. Previous measurements of $\phi_{s}$ by the CDF and D0 collaborations [37, 38, gave tantalising indications of non-SM physics. Recent results from LHCb using resonant and non-resonant $B_{s}^{0} \rightarrow J / \psi K^{+} K^{-}$and $B^{0} \rightarrow J / \psi \pi^{+} \pi^{-}$decays 39] and the flavour-tagged analysis from ATLAS [40, shown in Fig. 8, show that no large new physics effects are observed and that it is now crucial to improve the experimental precision. Another interesting development is the first measurement of the CP-violating phase $\phi_{s}$ using the rare penguin process $B_{s}^{0} \rightarrow \phi \phi$ by the $\mathrm{LHCb}$ collaboration [1]. The comparison of $\phi_{s}$ measured using tree-level and penguin processes may help to clarify a long-term observed difference in $\sin 2 \beta$ measured using $b \rightarrow c \bar{c} s$ and $b \rightarrow c \bar{c} d$ transitions [42].

Very recently, the $\mathrm{LHCb}$ collaboration has discovered $\mathrm{CP}$ violation in the $B_{s}^{0}$ system, using the $B_{s}^{0} \rightarrow K^{-} \pi^{+}$decay mode [43, as shown in Fig. 9] The observed CP violation leads to a $\mathrm{CP}$ asymmetry of $A_{\mathrm{CP}}\left(B_{s}^{0} \rightarrow K^{-} \pi^{+}\right)=0.27 \pm 0.04$ (stat.) \pm 0.01 (syst.) in the decay amplitude, assuming the absence of $\mathrm{CP}$ violation in the mixing. Large $\mathrm{CP}$ violation in the decay (a.k.a. direct CP violation) has also been observed at the LHC in charged $B$ decays. In particular, the Dalitz plot distributions of $B^{-} \rightarrow h^{+} h^{-} h^{-}$decays, where $h$ is a charged pion or kaon, show significant CP violation in some regions [44].

A major responsibility of the $\mathrm{LHCb}$ experiment is to provide a solid benchmark of the SM picture of quark flavour interactions against which new physics contributions can be judged. An important aspect of this exercise is the precise determination of the CKM unitarity triangle angle $\gamma$, both in tree-level decays, such as $B^{-} \rightarrow D h^{-}$, and in loop-dominated modes, such as $B_{(s)}^{0} \rightarrow h^{+} h^{-}$. A recent combination of three $\mathrm{LHCb}$ $B^{-} \rightarrow D h^{-}$measurements, where the $D$ is reconstructed in two-, three- and four-body final states provides a measurement of $\gamma=67 \pm 12^{\circ}$ [45. This is the most precise measurement of $\gamma$ from a single experiment to date.

Since both mixing-induced and direct CP violation are now established in the $B$ system, the question remains as to whether $\mathrm{CP}$ violation also exists in $B$ mixing. 


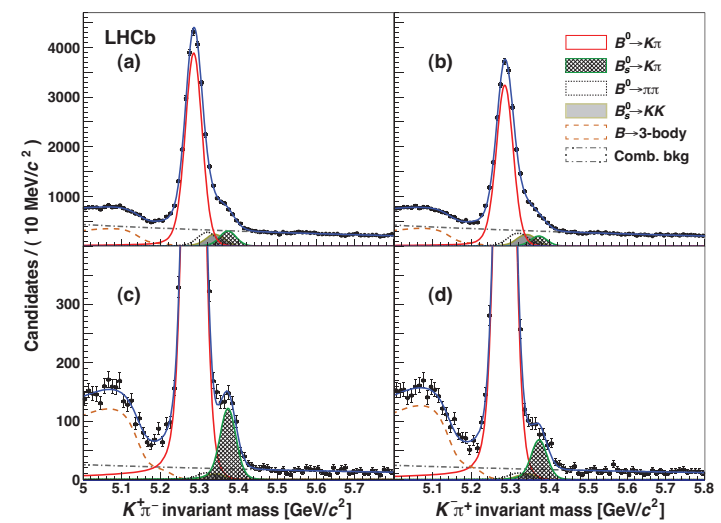

Figure 9. Invariant mass spectra obtained using the event selection adopted for the best sensitivity on (a), (b) $A_{\mathrm{CP}}\left(B^{0} \rightarrow K^{+} \pi^{-}\right)$and (c), (d) $A_{\mathrm{CP}}\left(B_{s}^{0} \rightarrow K^{-} \pi^{+}\right)$[3]. Panels (a) and (c) represent the $K^{+} \pi^{-}$invariant mass, whereas panels (b) and (d) represent the $K^{-} \pi^{+}$invariant mass.

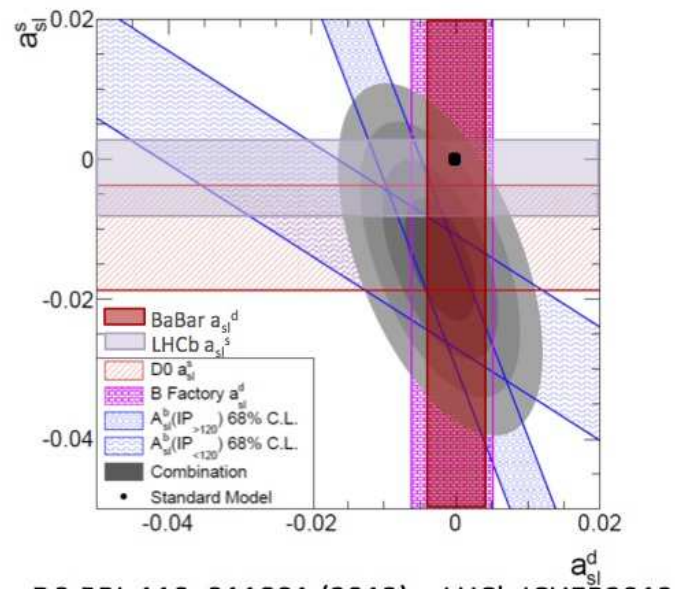

Figure 10. Measurements of the CP-violating asymmetry for semi-leptonic $B^{0}$ versus $B_{s}^{0}$ decays from D0 [46-48], LHCb [50] and BaBar [49].

Measurements by the D0 collaboration of the dimuon charge asymmetry in semileptonic $B$ decays, which is related to the phases in the mass and lifetime matrices for $B^{0}$ and $B_{s}^{0}$ mesons $\phi_{q}=\arg \left(M_{12} / \Gamma_{12}\right)$, show a $3.9 \sigma$ deviation from the SM expectation [46]. Further measurements from the D0 [47, 48], BaBar [49] and LHCb [50] collaborations, which separate out the contributions from $B^{0}$ and $B_{s}^{0}$ decays, have lowered the significance to 2.4 $\sigma$. A summary of all the measurements is shown in Fig. 10,

The final conundrum in the area of $\mathrm{CP}$ violation, is whether $\mathrm{CP}$ violation exists in the charm sector. In 2012, LHCb published a measurement of the difference in $\mathrm{CP}$ asymmetries between $D^{0} \rightarrow K^{+} K^{-}$and $D^{0} \rightarrow \pi^{+} \pi^{-}$decays, $\Delta A_{\mathrm{CP}}=$ $A_{\mathrm{CP}}\left(K^{+} K^{-}\right)-A_{\mathrm{CP}}\left(\pi^{+} \pi^{-}\right)$, using prompt $D^{0}$ mesons from $D^{*+} \rightarrow D^{0} \pi^{+}$decays [51]. The measurement deviated from the extremely small SM expectation by 3.5 standard deviations. The result was subsequently confirmed by the CDF [52] and Belle [53] collaborations. New results from LHCb updating the prompt analysis [54] and using semileptonic $B$ decays [55] reduce the significance. A compendium of the current status 


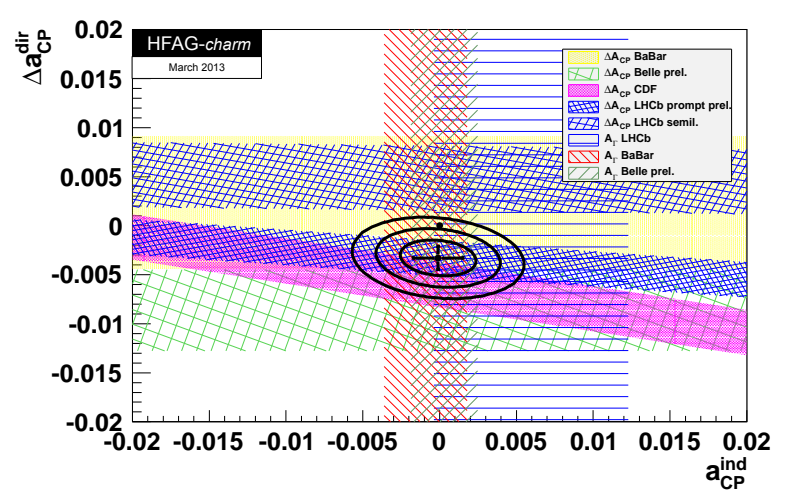

Figure 11. Recent compilation of experimental results by the HFAG [42] of indirect versus direct $\mathrm{CP}$-violating asymmetry observables in the $D^{0}$ system.

given by the HFAG [42] is shown in Fig. 11. There is a clear need for more experimental input to this picture, including improved statistical precision and measurements in other $D$ decay modes expecially for those that separate the contributions from direct and indirect $\mathrm{CP}$ violation.

\section{Rare decays}

Searching for very rare $B$ and $D$ decay modes is of paramount importance in heavy flavour physics, since many new physics models predict observables that differ significantly from the SM expectations.

One of the most Nobel [sic] places to search for new physics and test the hypothesis of $\mathrm{MFV}$ is in $B_{(s)}^{0} \rightarrow \mu^{+} \mu^{-}$decays. The $B_{s}^{0} \rightarrow \mu^{+} \mu^{-}$decay is a pure loop, helicity and CKM suppressed, process whose SM branching fraction, $B\left(B_{s}^{0} \rightarrow \mu^{+} \mu^{-}\right)_{S M}=$ $(3.54 \pm 0.30) \times 10^{-9}$ [56] (updated in Ref. [57]), is known to very good precision. However, large enhancements are possible in many variants of supersymmetry and alternative new physics models. Several impressive limits have been set by the LHC and Tevatron experiments and the first $3.5 \sigma$ evidence for the decay has been seen by the $\mathrm{LHCb}$ collaboration [57. The observation, shown in Fig. 12, leads to a measured branching fraction of $B\left(B_{s}^{0} \rightarrow \mu^{+} \mu^{-}\right)=\left(3.2_{-1.2}^{+1.4}(\text { stat })_{-0.3}^{+0.5}\right.$ (syst $\left.)\right) \times 10^{-9}$, which is consistent with the SM expectation and rules out the minimal supersymmetric extension to the SM with a light pseudoscalar Higgs at large $\tan \beta$. It is now vital to measure $B\left(B_{s}^{0} \rightarrow \mu^{+} \mu^{-}\right)$at the level of the theoretical uncertainty (a few $\times 10^{-10}$ ) and to measure it's relationship with $B^{0} \rightarrow \mu^{+} \mu^{-}$to test the MFV hypothesis and distinguish between new physics models, such as those with partial compositeness [58].

The rare decay mode $B^{0} \rightarrow K^{* 0} \mu^{+} \mu^{-}$provides a powerful approach to search for physics beyond the SM and to study it's helicity structure. The decay mode is also both experimentally and theoretically clean, especially at low dimuon mass-squared, and offers many measureable observables (rates, angular distributions and $\mathrm{CP}$ asymmetries) that are sensitive to new physics contributions. The flagship measurement is the forward-backward asymmetry, $A_{F B}$, defined by the direction of the postively-charged 


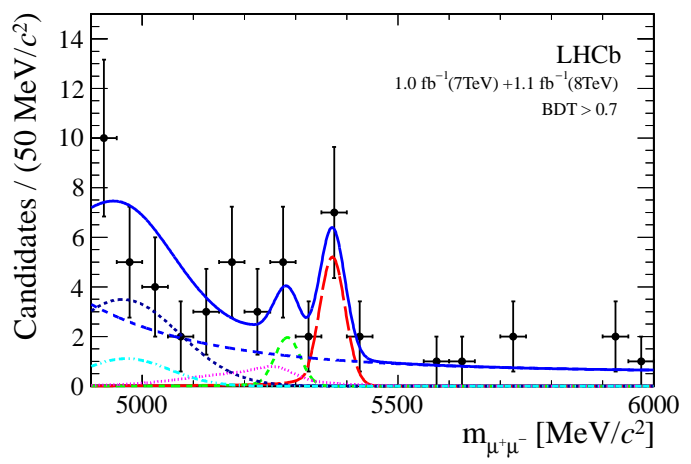

Figure 12. Invariant mass distribution of $B_{s}^{0} \rightarrow \mu^{+} \mu^{-}$candidates (black dots) [57. The result of the fit is overlaid (blue solid line) and the different components detailed: $B_{s}^{0} \rightarrow \mu^{+} \mu^{-}$(red long dashed curve), $B^{0} \rightarrow \mu^{+} \mu^{-}$(green medium dashed curve), $B_{(s)}^{0} \rightarrow h^{+} h^{-}$(pink dotted curve), $B^{0} \rightarrow \pi^{-} \mu^{+} \nu_{\mu}$ (black short dashed curve), and $B^{0(+)} \rightarrow \pi^{0(+)} \mu^{+} \mu^{-}$(light blue dash-dotted curve), and the combinatorial background (blue medium dashed).
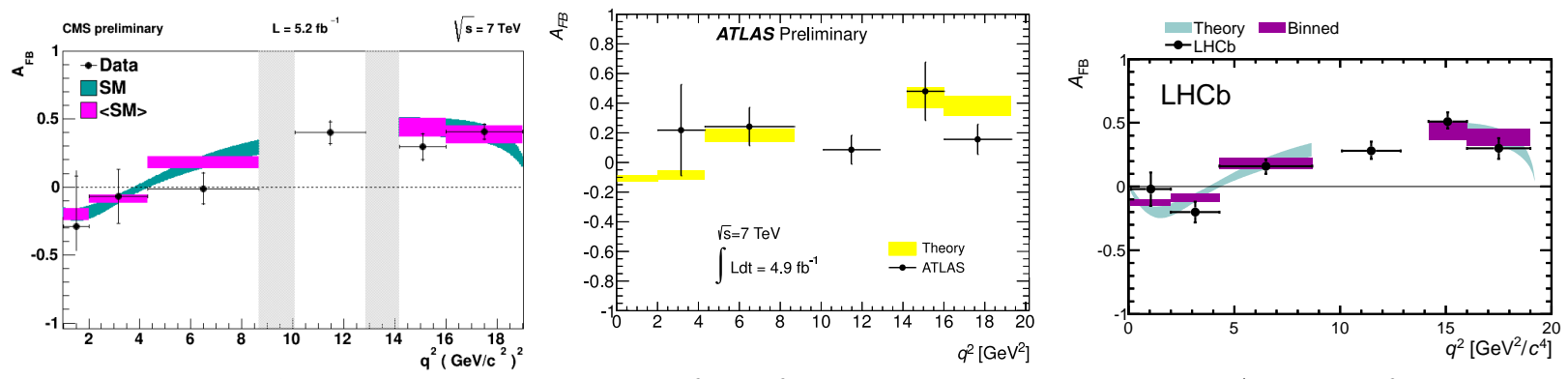

Figure 13. Measurements of the forward-backward asymmetry $A_{F B}$ as a function of the dimuon mass-squared in $B^{0} \rightarrow K^{*} \mu^{+} \mu^{-}$decays from (left) CMS [60], (centre) ATLAS [59] and (right) LHCb 61].

muon. Recent results from ATLAS [59, CMS [60 and LHCb 61], shown in Fig. 13], show consistency with the SM expectation. Of particular interest is the forward-backward asymmetry zero-crossing point, measured by LHCb to be $q_{0}^{2}=4.9 \pm 0.9 \mathrm{GeV}^{2} / c^{4}$, once again consistent with the SM theory predictions ranging between 3.9 and $4.4 \mathrm{GeV}^{2} / c^{4}$ with $10 \%$ uncertainties. The HFAG average of all previous measurements is now eagerly awaited and studies of other observables, which will offer new opportunities to distinguish between the new physics models, are now starting to be made.

The search for rare $D$ decays at the LHC also offers many opportunities to search for new phenomena beyond the SM. The $D^{0} \rightarrow \mu^{+} \mu^{-}$decay, dominated by long-distance contributions from the $\gamma \gamma$ intermediate state, is helicity and GIM suppressed, and has an expected SM branching fraction $B\left(D^{0} \rightarrow \mu^{+} \mu^{-}\right)<6 \times 10^{-11}$. A recent search by the LHCb collaboration, shown in Fig. 14(left), results in an upper limit on the branching fraction of $B\left(D^{0} \rightarrow \mu^{+} \mu^{-}\right)<6.2 \times 10^{-9}$ at $90 \%$ confidence level [62, two orders of magnitude lower than previous searches.

The LHCb collaboration has also searched for flavour-changing neutral current 

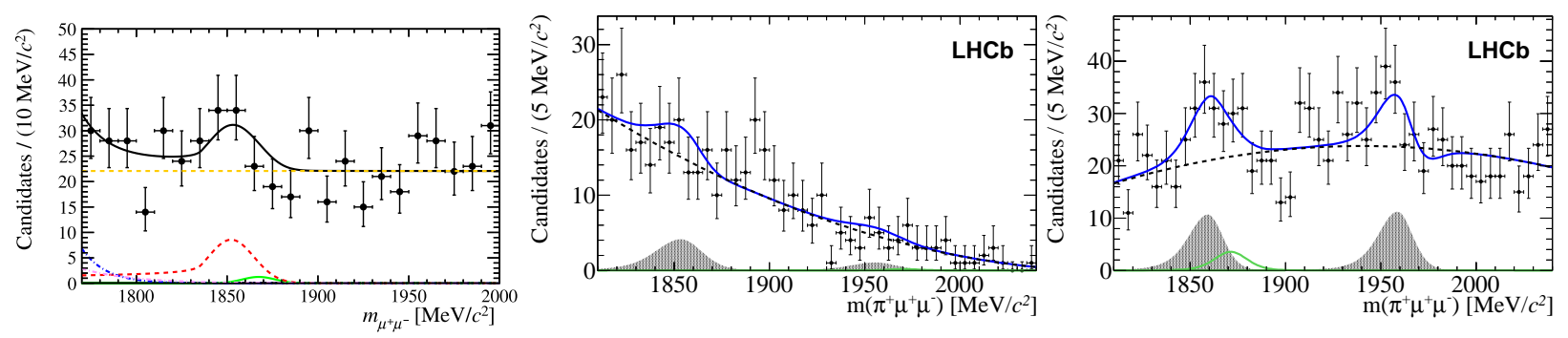

Figure 14. Invariant mass distributions for (left) $D^{0} \rightarrow \mu^{+} \mu^{-}$candidates [62, and (centre) low dimuon mass and (right) high dimuon mass $D_{(s)}^{+} \rightarrow \pi^{+} \mu^{+} \mu^{-}$ candidates 63] from $\mathrm{LHCb}$.

decays $D_{(s)}^{+} \rightarrow \pi^{+} \mu^{+} \mu^{-}$, with expected branching fractions $B\left(c \rightarrow u \mu^{+} \mu^{-}\right)_{S M}=$ $(1-3) \times 10^{-9}$, and their like-sign dimuon counterparts $D_{(s)}^{+} \rightarrow \pi^{+} \mu^{+} \mu^{+}$that are a signature for Majorana neutrinos 63]. The results show a resonance structure, with the expected $\eta, \rho / \omega$ and $\phi$ peaks in the resonance regions, and no signal peaks beyond background expectations in the non-resonance regions (Figure 14(centre) and (right)). The limits set indicate that an observation of this class of decays will soon be within reach.

\section{Future prospects and summary}

Heavy flavour physics at the LHC has blossomed over the past two years. In particular, the LHCb experiment has made the most tremendous start in producing many worldclass measurements and exploring new avenues that may reveal new physics in heavy flavour observables. The short term prospects are excellent. There is much data taken prior to and still to be collected after the first LHC shutdown period, which will be analysed by the experiments.

The second half of this decade will see the transition to the next generation of experiments. The LHCb upgrade will be installed in the second LHC long-shutdown period (2018/19) and will provide a detector capable of running at an operational luminosity well above $10^{33} \mathrm{~cm}^{-2} \mathrm{~s}^{-1}$ 64.

After the upgrade, $\mathrm{LHCb}$ aims to collect more than $50 \mathrm{fb}^{-1}$ of integrated luminosity over a period of 10 years. With this amount of data and that collected by ATLAS/CMS, there are fantastic prospects for heavy flavour physics at the LHC. The LHCb upgrade physics programme is extensive and includes many key apects: precision measurements of CP-violating phases in the $B_{s}^{0}$ system, in particular in the pure penguin-dominated process $B_{s}^{0} \rightarrow \phi \phi$; a factor of 10 reduction in the uncertainty on the CKM angle $\gamma$; a measurement of the ratio of the very rare $B_{s}^{0} \rightarrow \mu^{+} \mu^{-}$decay rate to that of the superrare $B^{0} \rightarrow \mu^{+} \mu^{-}$decay; the study of $\mathrm{CP}$ violation in the charm sector; $t \bar{t}$ production asymmetries; and other unique measurements in topics beyond heavy flavour. There is no doubt that the tale of heavy flavour at the LHC will continue for many years to come. 


\section{My questions and acknowledgements}

As part of this wonderful Nobel Symposium, we were asked to prepare some questions for discussion. Here are my questions and some of my tentative answers, for which I take total responsibility...

(i) If the LHCb upgrade does not reveal new physics by the end of the 2020's, what is the fate of flavour physics?

If a high energy pp collider is operational in the future, it is hard to believe that we would not want to take advantage of the copious production of heavy flavours. Or, in other words, it is hard to imagine that there will be proof that New Physics (whether seen or still hypothesized) leaves no signature in the heavy flavour sector. Once the upgraded $\mathrm{LHCb}$ detector has demonstrated that it's $40 \mathrm{MHz}$ read-out and triggering works, it will be natural to think about how the luminosity could be raised further.

(ii) At the moment the heavy flavour community is pursuing two roads to discovery, $\mathrm{CP}$ violation and rare decays. Should we contemplate, for example a "rare decay only" experiment?

One of the main issues for the LHCb upgrade, and most likely for any other "generic" heavy flavour experiment at similar or higher luminosities, is the massive data rate, which is a consequence of the broad physics programme. So it seems natural to ask whether it would make sense to design an experiment that instead is focussed on one or a few specific channels. For example, to measure the ratio of branching fractions $\mathcal{B}\left(B^{0} \rightarrow \mu^{+} \mu^{-}\right) / \mathcal{B}\left(B_{s}^{0} \rightarrow \mu^{+} \mu^{-}\right)$at a few percent precision would need approximately two orders of magnitude more data that we can get from the LHCb upgrade - quite possibly the only way to get there, say within 25 years from now, is with a dedicated experiment. Although such an experiment is not the preferred option at this time, it seems valid to consider it.

(iii) What ultimate precision on $\mathrm{CP}$ violation and rare decay observables should a quark flavour physics experiment aspire to (i.e. is there a need to go well beyond SM theory uncertainties)?

There are several flavour observables with theoretical uncertainties much smaller than even those that can be acheived by the LHCb upgrade. So for the foreseeable future, I think we just have to aim for the best precision we can achieve.

(iv) We do not seem to be making much progress towards a "theory of flavour" (i.e. an understanding of the patterns in, and relations (if any) between, the quark and lepton masses and mixing. Are different experimental and/or theoretical approaches needed to address this question?

Probably not, but I'd be glad to hear if people think otherwise!

Finally, I would like to thank my colleagues on LHCb for all the tremendous effort in producing many beautiful results and as we look forward to a very fruitful future. 
In particular, I would like to thank my colleagues in Cambridge and Tim Gershon, Pierluigi Campana and Tatsuya Nakada for their help in preparation of this review. I would also like to thank the organizing committee for inviting me and making this Nobel Symposium a very enjoyable experience.

\section{References}

[1] M. Kobayashi and T. Maskawa, CP violation in the renormalizable theory of weak interaction, Prog. Theor. Phys. 49 (1973) 652 N. Cabibbo, Unitary symmetry and leptonic decays, Phys. Rev. Lett. 10 (1963) 531

[2] CKMfitter group, J. Charles et al., CP violation and the CKM matrix: assessing the impact of the asymmetric B factories, Eur. Phys. J. C41 (2005) 1, arXiv:hep-ph/0406184 updated results and plots available at: http://ckmfitter.in2p3.fr

[3] UTfit collaboration, M. Bona et al., The 2004 UTfit collaboration report on the status of the unitarity triangle in the standard model, JHEP 07 (2005) 028, arXiv:hep-ph/0501199 updated results and plots available at: http://www.utfit.org/UTfit/

[4] BaBar collaboration, B. Aubert et al., The BaBar detector: upgrades, operation and performance, arXiv: 1305.3560

[5] Belle collaboration, A. Abashian et al., The Belle detector, Nucl. Instrum. Meth. A479 (2002) 117.

[6] CDF collaboration, R. Blair et al., The CDF-II detector, FERMILAB-PUB-96-390-E

[7] D0 collaboration, V. Abazov et al., The upgraded Do detector, Nucl. Instrum. Meth. A565 (2006) 463, arXiv:physics/0507191.

[8] J. Laiho, E. Lunghi, and R. S. Van de Water, Lattice QCD inputs to the CKM unitarity triangle analysis, Phys. Rev. D81 (2010) 034503 arXiv:0910.2928 updated results and plots available at: http://www. latticeaverages.org/

[9] T. Hurth and F. Mahmoudi, The Minimal Flavour Violation benchmark in view of the latest LHCb data, Nucl. Phys. B865 (2012) 461, arXiv:1207.0688 and references therein.

[10] LHCb collaboration, A. A. Alves Jr. et al., The LHCb detector at the LHC, JINST 3 (2008) S08005

[11] ATLAS collaboration, G. Aad et al., The ATLAS experiment at the CERN Large Hadron Collider, JINST 3 (2008) S08003

[12] CMS collaboration, S. Chatrchyan et al., The CMS experiment at the CERN LHC, JINST 3 (2008) S08004.

[13] ATLAS Collaboration, G. Aad et al., Measurement of the differential cross-section of $B^{+}$meson production in pp collisions at $\sqrt{s}=7$ TeV at ATLAS, arXiv:1307.0126

[14] LHCb collaboration, R. Aaij et al., Prompt charm production in pp collisions at $\sqrt{s}=7 \mathrm{TeV}$, Nucl. Phys. B871 (2013) 1, arXiv:1302.2864

[15] M. Cacciari et al., Theoretical predictions for charm and bottom production at the LHC, JHEP 1210 (2012) 137, arXiv:1205.6344.

[16] LHCb collaboration, R. Aaij et al., Production of $J / \psi$ and $\Upsilon$ mesons in pp collisions at $\sqrt{s}=$ $8 \mathrm{TeV}$, JHEP 06 (2013) 64 arXiv: 1304.6977.

[17] CMS Collaboration, S. Chatrchyan et al., Measurement of the $\Upsilon(1 S), \Upsilon(2 S)$ and $\Upsilon(3 S)$ polarizations in pp collisions at $\sqrt{s}=7$ TeV, Phys. Rev. Lett. 110 (2013) 081802 arXiv: 1209.2922

[18] ATLAS collaboration, G. Aad et al., Observation of a new $\chi_{b}$ state in radiative transitions to $\Upsilon(1 S)$ and $\Upsilon(2 S)$ at $A T L A S$, Phys. Rev. Lett. 108 (2012) 152001, arXiv:1112.5154.

[19] CMS collaboration, S. Chatrchyan et al., Observation of a new $\Xi_{b}$ baryon, Phys. Rev. Lett. 108 (2012) 252002, arXiv:1204.5955.

[20] LHCb collaboration, R. Aaij et al., Observation of excited $\Lambda_{b}^{0}$ baryons, Phys. Rev. Lett. 109 (2012) 172003 , arXiv:1205.3452. 
[21] LHCb collaboration, R. Aaij et al., Precision measurements of D meson mass differences, JHEP 06 (2013) 65, arXiv:1304.6865.

[22] CMS collaboration, S. Chatrchyan et al., Measurement of the $\Lambda_{b}^{0}$ lifetime in pp collisions at $\sqrt{s}=7$ TeV, JHEP 07 (2013) 163 arXiv:1304.7495.

[23] Particle Data Group, J. Beringer et al., Review of particle physics, Phys. Rev. D86 (2012) 010001

[24] Belle collaboration, S. Choi et al., Observation of a narrow charmonium-like state in exclusive $B^{ \pm} \rightarrow K^{ \pm} \pi^{+} \pi^{-} J / \psi$ decays, Phys. Rev. Lett. 91 (2003) 262001, arXiv:hep-ex/0309032.

[25] LHCb collaboration, R. Aaij et al., Observation of $X(3872)$ production in pp collisions at $\sqrt{s}=7 \mathrm{TeV}$, Eur. Phys. J. C72 (2011) 1972, arXiv:1112.5310

[26] CMS collaboration, S. Chatrchyan et al., Measurement of the X(3872) production cross-section via decays to $J / \psi \pi \pi$ in pp collisions at $\sqrt{s}=7 \mathrm{TeV}$, JHEP 1304 (2013) 154, arXiv:1302.3968

[27] CDF collaboration, A. Abulencia et al., Analysis of the quantum numbers $J^{P C}$ of the X(3872), Phys. Rev. Lett. 98 (2007) 132002, arXiv: hep-ex/0612053

[28] LHCb collaboration, R. Aaij et al., Determination of the X(3872) quantum numbers, Phys. Rev. Lett. 110 (2013) 222001, arXiv:1302.6269

[29] CDF collaboration, T. Aaltonen et al., Evidence for a narrow near-threshold structure in the $J / \psi \phi$ mass spectrum in $B^{+} \rightarrow J / \psi \phi K^{+}$decays, Phys. Rev. Lett. 102 (2009) 242002 arXiv:0903.2229

[30] LHCb collaboration, R. Aaij et al., Search for the $X(4140)$ state in $B^{+} \rightarrow J / \psi \phi K^{+}$decays, Phys. Rev. D85 (2012) 091103(R), arXiv:1202.5087

[31] CMS collaboration, S. Chatrchyan et al., Observation of structures in $J / \psi \phi$ spectrum in exclusive $B^{+} \rightarrow J / \psi \phi K^{+}$decays at $7 \mathrm{TeV}$, CMS-BPH-11-026

[32] LHCb collaboration, R. Aaij et al., Observation of $D^{0}-\bar{D}^{0}$ oscillations, Phys. Rev. Lett. 110 (2013) 101802, arXiv:1211.1230

[33] LHCb collaboration, R. Aaij et al., Measurement of the $B^{0}-\bar{B}^{0}$ oscillation frequency $\Delta m_{d}$ with the decays $B^{0} \rightarrow D^{-} \pi^{+}$and $B^{0} \rightarrow J / \psi K^{* 0}$, Phys. Lett. B719 (2013) 318, arXiv:1210.6750.

[34] LHCb collaboration, R. Aaij et al., Precision measurement of the $B_{s}^{0}-\bar{B}_{s}^{0}$ oscillation frequency $\Delta m_{s}$ in the decay $B_{s}^{0} \rightarrow D_{s}^{+} \pi^{-}$, New J. Phys. 15 (2013) 053021 arXiv:1304.4741.

[35] BaBar collaboration, B. Aubert et al., Measurement of time-dependent CP asymmetry in $B^{0} \rightarrow c \bar{c} K^{(*) 0}$ decays, Phys. Rev. D79 (2009) 072009 arXiv:0902.1708 I. Adachi et al., Precise measurement of the CP violation parameter $\sin 2 \phi_{1}$ in $B^{0} \rightarrow(c \bar{c}) K^{0}$ decays, Phys. Rev. Lett. 108 (2012) 171802, arXiv:1201.4643,

[36] LHCb collaboration, R. Aaij et al., Measurement of the $B_{s}^{0} \rightarrow J / \psi K_{\mathrm{S}}^{0}$ effective lifetime, Nucl. Phys. B873 (2013) 275, arXiv:1304.4500

[37] CDF collaboration, T. Aaltonen et al., Measurement of the bottom-strange meson mixing phase in the full CDF data set, Phys. Rev. Lett. 109 (2012) 171802, arXiv:1208.2967

[38] D0 collaboration, V. M. Abazov et al., Measurement of the CP-violating phase $\phi_{s}^{J / \psi \phi}$ using the flavor-tagged decay $B_{s}^{0} \rightarrow J / \psi \phi$ in $8 f^{-1}$ of $p \bar{p}$ collisions, Phys. Rev. D85 (2012) 032006 arXiv:1109.3166

[39] LHCb collaboration, R. Aaij et al., Measurement of CP-violation and the $B_{s}^{0}$-meson decay width difference with $B_{s}^{0} \rightarrow J / \psi K^{+} K^{-}$and $B_{s}^{0} \rightarrow J / \psi \pi^{+} \pi^{-}$decays, Phys. Rev. D87 (2013) 112010 arXiv: 1304.2600

[40] ATLAS collaboration, Flavour-tagged time-dependent angular analysis of the $B_{s} \rightarrow J / \psi \phi$ decay and extraction of $\Delta \Gamma$ and the weak phase $\phi_{s}$ in ATLAS, ATLAS-CONF-2013-039

[41] LHCb collaboration, R. Aaij et al., First measurement of the CP-violating phase in $B_{s}^{0} \rightarrow \phi \phi$ decays, Phys. Rev. Lett. 110 (2013) 241802, arXiv:1303.7125.

[42] Heavy Flavor Averaging Group, Y. Amhis et al., Averages of b-hadron, c-hadron, and $\tau$ lepton properties as of early 2012, arXiv:1207.1158, updated results and plots available at http://www.slac.stanford.edu/xorg/hfag/

[43] LHCb collaboration, R. Aaij et al., First observation of $C P$ violation in the decays of $B_{s}^{0}$ mesons, Phys. Rev. Lett. 110 (2013) 221601, arXiv:1304.6173 
[44] LHCb collaboration, R. Aaij et al., Measurement of CP violation in the phase space of $B^{ \pm} \rightarrow$ $K^{ \pm} \pi^{+} \pi^{-}$and $B^{ \pm} \rightarrow K^{ \pm} K^{+} K^{-}$, Phys. Rev. Lett. 111 (2013) 101801, arXiv:1306.1246

[45] LHCb collaboration, R. Aaij et al., A measurement of $\gamma$ from a combination of $B^{ \pm} \rightarrow D h^{ \pm}$ analyses, arXiv:1305.2050, to appear in Phys. Lett. B; LHCb collaboration, A measurement of $\gamma$ from a combination of $B^{ \pm} \rightarrow D K^{ \pm}$analyses including first results using $2 \mathrm{fb}^{-1}$ of 2012 data, LHCb-CONF-2013-006

[46] D0 collaboration, V. M. Abazov et al., Measurement of the anomalous like-sign dimuon charge asymmetry with $9 \mathrm{fb}^{-1}$ of $p \bar{p}$ collisions, Phys. Rev. D84 (2011) 052007, arXiv:1106.6308

[47] D0 collaboration, V. M. Abazov et al., Measurement of the semileptonic charge asymmetry in $B^{0}$ meson mixing with the D0 detector, Phys. Rev. D86 (2012) 072009, arXiv:1208.5813

[48] D0 collaboration, V. Abazov et al., Measurement of the semileptonic charge asymmetry using $B_{s}^{0} \rightarrow D_{s} \mu X$ decays, Phys. Rev. Lett. 110 (2013) 011801 arXiv:1207.1769

[49] BaBar collaboration, J. Lees et al., Search for $C P$ violation in $B^{0}-\overline{B^{0}}$ mixing using partial reconstruction of $B^{0} \rightarrow D^{*-} X l^{+} \nu$ and a kaon tag, arXiv:1305.1575.

[50] LHCb collaboration, R. Aaij et al., Measurement of the flavour-specific CP-violating asymmetry $a_{\mathrm{sl}}^{s}$ in $B_{s}^{0}$ decays, arXiv:1308.1048, submitted to Phys. Lett. B.

[51] LHCb collaboration, R. Aaij et al., Evidence for CP violation in time-integrated $D^{0} \rightarrow h^{-} h^{+}$decay rates, Phys. Rev. Lett. 108 (2012) 111602 arXiv:1112.0938

[52] CDF collaboration, T. Aaltonen et al., Measurement of the difference of CP-violating asymmetries in $D^{0} \rightarrow K^{+} K^{-}$and $D^{0} \rightarrow \pi^{+} \pi^{-}$decays at $C D F$, Phys. Rev. Lett. 109 (2012) 111801 arXiv:1207.2158

[53] Belle collaboration, B. R. Ko, CP violation and mixing in the charm sector at Belle, and current HFA $G$ averages, arXiv: 1212.5320

[54] LHCb collaboration, A search for time-integrated $C P$ violation in $D^{0} \rightarrow K^{-} K^{+}$and $D^{0} \rightarrow \pi^{-} \pi^{+}$ decays, LHCb-CONF-2013-003

[55] LHCb collaboration, R. Aaij et al., Search for direct $C P$ violation in $D^{0} \rightarrow h^{-} h^{+}$modes using semileptonic $B$ decays, Phys. Lett. B723 (2013) 33, arXiv:1303.2614

[56] A. J. Buras, J. Girrbach, D. Guadagnoli, and G. Isidori, On the standard model prediction for $\mathcal{B}\left(B_{s, d} \rightarrow \mu^{+} \mu^{-}\right)$, Eur. Phys. J. C72 (2012) 2172 arXiv:1208.0934

[57] LHCb collaboration, R. Aaij et al., First evidence for the decay $B_{s}^{0} \rightarrow \mu^{+} \mu^{-}$, Phys. Rev. Lett. 110 (2013) 021801, arXiv:1211.2674

[58] D. M. Straub, Anatomy of flavour-changing $Z$ couplings in models with partial compositeness, JHEP 1308 (2013) 108, arXiv:1302.4651.

[59] ATLAS collaboration, Angular analysis of $B_{d} \rightarrow K^{* 0} \mu^{+} \mu^{-}$with the ATLAS experiment, ATLAS-CONF-2013-038

[60] CMS collaboration, Angular analysis and branching ratio measurement of the decay $B^{0} \rightarrow$ $K^{* 0} \mu^{+} \mu^{-}$, CMS-PAS-BPH-11-009

[61] LHCb collaboration, R. Aaij et al., Differential branching fraction and angular analysis of the decay $B^{0} \rightarrow K^{* 0} \mu^{+} \mu^{-}$, JHEP 08 (2013) 131, arXiv:1304.6325.

[62] LHCb collaboration, R. Aaij et al., Search for the rare decay $D^{0} \rightarrow \mu^{+} \mu^{-}$, Phys. Lett. B725 (2013) 15, arXiv:1305.5059

[63] LHCb collaboration, R. Aaij et al., Search for $D_{(s)}^{+} \rightarrow \pi^{+} \mu^{-} \mu^{-}$and $D_{(s)}^{+} \rightarrow \pi^{-} \mu^{+} \mu^{+}$decays, Phys. Lett. B724 (2013) 203 arXiv:1304.6365.

[64] LHCb collaboration, Letter of Intent for the LHCb Upgrade, CERN-LHCC-2011-001, LHCC-I-018 LHCb collaboration, I. Bediaga et al., Framework TDR for the LHCb Upgrade: Technical Design Report, CERN-LHCC-2012-007, LHCb-TDR-12. 